\title{
Effects of an intensive behavioral weight loss intervention consisting of caloric restriction with or without physical activity on common carotid artery remodeling in severely obese adults
}

\author{
Jennifer N. Cooper, MSa, Mindy L. Columbus, MPHa, Kelly J. Shields, PhDa, Julius \\ Asubonteng, MS ${ }^{a}$, Michelle L. Meyer, PhD ${ }^{b}$, Kim Sutton-Tyrrell, DrPH ${ }^{a}$, Bret H. Goodpaster, \\ $\mathbf{P h D}^{\mathrm{c}}$, James P. DeLany, $\mathrm{PhD}^{\mathrm{c}}$, John M. Jakicic, $\mathbf{P h D}^{\mathrm{d}}$, and Emma Barinas-Mitchell, $\mathbf{P h D}^{\mathrm{a}}$

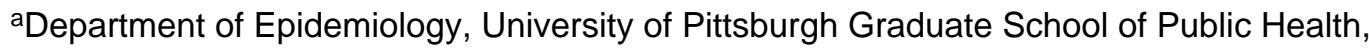 \\ Pittsburgh, Pennsylvania \\ bDepartment of Epidemiology, University of North Carolina at Chapel Hill, Gillings School of \\ Global Public Health, Chapel Hill, North Carolina \\ 'Division of Endocrinology and Metabolism, University of Pittsburgh School of Medicine, \\ Pittsburgh, Pennsylvania \\ dPhysical Activity and Weight Management Research Center, Department of Health and Physical \\ Activity, University of Pittsburgh, Pittsburgh, Pennsylvania
}

\section{Abstract \\ Objective-Obesity increases cardiovascular disease risk and adversely affects vascular structure and function. Few studies have evaluated the vascular effects of non-surgical weight reduction in the severely obese. We hypothesized that weight loss and improvements in cardiometabolic factors would reduce common carotid artery intima-media thickness (CIMT) and inter-adventitial diameter (AD) in severely obese adults.}

\begin{abstract}
Methods-We performed carotid ultrasound and measured cardiometabolic factors in 90 severely obese participants (body mass index (BMI) $\geq 35 \mathrm{~kg} / \mathrm{m}^{2}$, age 30-55) at baseline and 6 months in a randomized clinical trial of dietary intervention with $(n=45)$ or without $(n=45)$ physical activity.
\end{abstract}

Results-The achieved weight loss (mean $=8 \%$ ) did not differ significantly by intervention group $(P=0.10)$ and resulted in a $0.07 \mathrm{~mm}$ mean decrease in $\mathrm{AD}(P=0.001)$. AD change was positively

(C) 2012 Elsevier Inc. All rights reserved.

Corresponding author: Jennifer Cooper, MS University of PittsburghGraduateSchool of Public Health Department of Epidemiology EpidemiologyDataCenter 130 DeSoto St. Pittsburgh, PA, USA 15261 Phone: (412) 624-4293 Fax: (412) 624-3775 jnn9@ pitt.edu. Publisher's Disclaimer: This is a PDF file of an unedited manuscript that has been accepted for publication. As a service to our customers we are providing this early version of the manuscript. The manuscript will undergo copyediting, typesetting, and review of the resulting proof before it is published in its final citable form. Please note that during the production process errors may be discovered which could affect the content, and all legal disclaimers that apply to the journal pertain.

Trial registration: clinicaltrials.gov Identifier: NCT00712127

Disclosure Dr. Jakicic serves on the scientific advisory board for Alere Well Being, serves as principal investigator on a research grant from BodyMedia, Inc. to the University of Pittsburgh, and has received an honorarium from Nestle for a research presentation. All other authors declare no conflict of interest.

Author Contributions The authors' contributions were as follows - JNC, MLC, KJS, JA, MLM, KS-T, EB-M: conceived the current hypothesis tested and wrote the initial draft of the manuscript; JNC, MLC, KJS, JA, MLM, EB-M: had full access to all of the data in the study and take responsibility for the integrity of the data; KS-T, EB-M, BHG, JPD, JMJ: conceived, designed, and conducted the clinical trial; JNC, KJS, JA, MLM: analyzed data; and all authors: interpreted data and critically revised the manuscript over successive drafts. 
correlated with changes in BMI, waist circumference, abdominal visceral and subcutaneous fat, and body fat mass, and $\mathrm{AD}$ decreased more in men ( $P<0.05$ for all). After multivariable adjustment, changes in BMI $(P=0.03)$ and abdominal subcutaneous fat $(P=0.04)$ were significant determinants of AD change. Although CIMT did not decrease significantly overall $(-0.008 \mathrm{~mm}$, $P=0.16$ ), individuals who lost at least $5 \%$ of their body weight experienced a significant mean reduction in CIMT of $0.02 \mathrm{~mm}(P=0.002)$. CIMT change was positively correlated with changes in BMI, waist circumference, fat-free mass, leptin, and insulin $(P<0.05$ for all). After multivariable adjustment, insulin reduction remained a significant determinant of CIMT decrease $(P=0.03)$.

Conclusion-A6 month intensive behavioral intervention can significantly reverse metabolic and vascular abnormalities in severely obese adults.

\section{Keywords}

Severe obesity; Carotid intima-media thickness; Carotid diameter; Lifestyle modification

\section{INTRODUCTION}

Obesity adversely affects vascular structure and function by increasing total blood volume and cardiac output, which can increase arterial wall stress, vascular smooth muscle cell proliferation, and vascular wall thickness and diameter . Weight loss reverses some of these adverse vascular changes and improves cardiovascular disease risk factors such as high blood pressure, dyslipidemia, insulin resistance, and elevated circulating levels of C-reactive proteinand adipokines. The fastest growing segment of the obese population is the severely obese, those with body mass index (BMI) $\geq 40 \mathrm{~kg} / \mathrm{m}^{2}$. In this population, weight loss may be achieved most efficiently through bariatric surgery due to the difficulties of achieving longterm success with lifestyle modifications . However, since only about $1 \%$ of the severely obese population receives bariatric surgery annually, non-surgical interventions warrant further consideration. Though there is skepticism regarding the ability of severely obese persons to achieve and sustain substantial non-surgical weight loss, there exists some evidence that intensive medical interventions can be effective .

Carotid intima-media thickness (CIMT) is a validated surrogate measure of subclinical atherosclerosis that is predictive of vascular events . Improvements in cardiovascular risk factors occurring with lifestyle modification are associated with favorable changes in CIMT . Carotid inter-adventitial diameter (AD) is another measure of arterial remodeling and predictsvascular events independent of CIMT.In large arteries, obesity is accompanied by diameterexpansion resulting from elevated circulating blood volume and pressure.This occurs concurrently with intima-media thickening, a process more reflective of atherosclerotic progression. This structural adaptive response to inflammatory, metabolic, and hemodynamic factors aims to maintain a balance between shear and tensile stresses in the vessel wall .

In the severely obese, bariatric surgery improves cardiovascular risk factors and results in the regression of CIMT , but questions remain as to whether clinically meaningful improvements in vascular structure can be achieved with lifestyle modification.Investigating whether intensive lifestyle interventions can effectively reduce cardiovascular disease risk in the severely obese will inform evidence-based guidelines allowing clinicians to provide more appropriate treatment recommendations to this population. We studied severely obese participants in a randomized clinical trial that assessed the impact of a dietary intervention with or without physical activity on weight loss, body composition, cardiometabolic factors, and common carotid artery structure. We hypothesized that weight loss and improvements in cardiometabolic factors, regardless of intervention assignment, would be associated with 
reductions in CIMT and AD over the course of the 6 month intervention. Additionally, we hypothesized that participants who received the physical activity intervention would achieve greater weight loss and greater reductions in CIMT and AD than participants who received only the dietary intervention.

\section{METHODS}

\section{Subject Recruitment / Randomization}

Recruitment and randomization methodshave been previously described. Briefly, participants were randomized into two study groups, with blocking according to sex, level of obesity (class II and III), and race/ethnicity (African American and white). Evaluators of all measures were blinded to group assignment. The study was reviewed and approved by the human ethics committees of the University of Pittsburgh with all participants providing written informed consent prior to enrollment.

\section{Inclusion/Exclusion Criteria}

Participants between the ages of 30 and 55 years were eligible if they were severely obese, defined in this study as a BMI between 35 and $39.9 \mathrm{~kg} / \mathrm{m}^{2}$ for class II obesity and $\geq 40 \mathrm{~kg} / \mathrm{m}^{2}$ for class III obesity. Participants had to be able to walk without assistance, commit to the schedule of intervention and assessment visits, and obtain medical clearance for intervention. Candidates were excluded if they had a history of cancer within the past 5 years, had a history of or were receiving current treatment for coronary artery disease, had enrolled within the past year in a formal weight reduction program, reported losing more than 5\% of their current body weight in the previous 6 months, had a history of bariatric surgery, uncontrolled hypertension, diabetes, or were pregnant during the previous 6 months.

\section{Treatment Groups}

All participants were randomized to a 12-month lifestyle intervention . One group was randomized to diet and physical activity for 12 months, while the other group had the identical dietary intervention but physical activity was delayed for 6 months. The primary goal of the trial was to examine the effect of an intensivelifestyle intervention on weight lossin severely obese adults and specifically to assess whether adoption of a physical activity program in addition to a dietary intervention would promote additionalweightlosscompared to a dietary intervention alone.Vascular measurements, which were not the primary measures of interest in this trial, were performed at only the baseline and 6 month visits.Thus, intervention group comparisons for the present study were diet plus physical activity ("Diet+PA") versus diet alone ("Diet"). The behavioral lifestyle intervention program was delivered with a combination of group, individual, and telephone contacts. During months 1 through 6, participants received 3 group meetings and 1 individual contact per month. All participants were prescribed a diet shown to produce a sustained 8-10\% weight loss within 12 months . Calculated energy intake was based on initial body weight and reduced to a target of between 1200 and $2100 \mathrm{kcal} /$ day. Targeted macronutrient composition was $20 \%$ to $30 \%$ fat, $50 \%$ to $55 \%$ carbohydrate, and $20 \%$ to $25 \%$ protein. To facilitate dietary compliance and improve weight loss, liquid and prepackaged meal replacements were provided at no cost for all but 1 meal per day during months 1 through 3 and for only 1 meal per day during months 4 through 6 of the intervention. Adherence to the dietary intervention was monitored by having participants record the time of meals as well as the type and caloric value of food consumed. For the Diet+PA intervention group, moderate intensity physical activity, similar to brisk walking, was recommended and progressed by $5-10 \mathrm{~min} /$ day in 4 week intervals to eventually reach 60 minutes, 5 days per week. To maximize adoption and maintenance of physical activity, participants were 
permitted to accumulate multiple 10-minute bouts of physical activity per day, were provided with a pedometer and step goals of more than 10,000 steps per day, and were instructed to self-monitor their physical activity in a weekly diary. To promote adherence, participants were provided with low-cost supplies related to the intervention (e.g. pedometer, exercise videos) and were eligible to periodically receive small financial incentives for adherence to the behavioral goals of the intervention.

\section{Study Measures}

Anthropometric / Physical—Body weight, height, and waist circumference were measured using standard protocols . Computed tomography (CT) scans (GE Light Speed CT scanner, GE Healthcare, Milwaukee, WI) were performed to quantify abdominal adipose tissue as previously described . Body fat and fat-free mass were determined either by dualenergy x-ray absorptiometry (DXA) (Lunar iDXA, GE Healthcare, Piscataway, NJ) or by air displacement plethysmography in 24 participants exceeding the weight capacity of the DXA scanner (136kg) (BOD POD, Life Measurement, Inc., Concord, CA). Blood pressure was measured with an automated system (Dinamap, GE Healthcare, Freiburg, Germany) after participants had sat quietly for 5 minutes. The average of the last 2 of 3 readings taken one minute apart was used.

\section{Serum measures}

Fasting blood samples were collected for the measurement of glucose, insulin, C-reactive protein, adiponectin, leptin, and lipids. Insulin resistance was calculated using the homeostatic model of insulin resistance assessment method. Adiponectin levels were assessed using xMAPTM bead-based technology (Luminex Corp., Austin, TX). Enzymelinked immunoassays were used to determine insulin (Millipore, Billerica, MA), leptin (R\&D Systems, Minneapolis, MN) and C-reactive protein (Helica Systems, Inc., Fullerton, CA). Total cholesterol, high density lipoprotein cholesterol, low density lipoprotein cholesterol, triglycerides, and glucose were determined using standard laboratory procedures as previously described .

\section{Physical Activity}

Objective measures of physical activity were quantified using multisensor physical activity monitors (Sensewear Pro3; BodyMedia, Pittsburgh, PA) worn between 7 and 11 consecutive days (mean(SD), 7.2(1.8) days; 23.1(0.6) hours/day) at baseline and 6 months.

\section{Carotid Ultrasound}

Primarily because of scheduling conflicts, only 90 of the 130 trial participants received a carotid ultrasound exam at both baseline and 6 months. CIMT was assessed by B-mode ultrasonography of the left and right common carotid arteries (CCA) with a Philips (Agilent) Imagepoint HX Ultrasound B-Mode duplex scanner (Philips, Andover, MA). Certified vascular sonographers from the University of Pittsburgh Ultrasound Research Laboratory performed the scans. Digitized images for reading were obtained from the left and right distal CCA, $1 \mathrm{~cm}$ proximal to the carotid bulb. CIMT measures were generated by electronically tracing the lumen-intima and media-adventitia interfaces across a $1 \mathrm{~cm}$ segment of both the near and far wall CCA. CCA inter-adventitial diameters were measured directly as the distance from the adventitial-medial interface on the near wall to the medialadventitial interface on the far wall at end-diastole across the same CCA segments. For analyses, the mean of the average CIMT and AD values were used. The reading software used was the AMS system developed by Dr. Thomas Gustavsson. Replicate scanning ( $n=14)$ yielded an intraclass correlation (ICC) of 0.82 for CIMT and 0.83 for $\mathrm{AD}$ and replicate readings $(n=20)$ yielded ICCs of 0.98 for CIMT and 0.95 for AD. 


\section{Statistical Analysis}

Descriptive statistics were calculated for demographic variables, cardiometabolic factors, CIMT, and AD. Differences in baseline characteristics between arms were assessed using ttests or Mann-Whitney U tests for continuous variables and the chi-square test or Fisher's exact test for categorical variables. The same analysis was performed to evaluate differences between trial participants with both baseline and 6 month carotid ultrasound scans $(\mathrm{n}=90)$ and trial participants missing carotid data at either time point $(\mathrm{n}=40)$ or at 6 months only $(n=19)$. Six month changes overall and within intervention arms were assessed using paired t-test or related samples Wilcoxon signed ranks test. Differences between arms were assessed using two-way analysis of variance (ANOVA) with adjustment for the sex difference between arms and log transformation of C-reactive protein and triglycerides. Pearson correlations were used to assess the associations between changes in CIMT or AD and changes in cardiometabolic factors.Mean changes in $\mathrm{AD}$ and CIMT were compared betweena priori subgroups based on baseline BMI or percent weightlossusing two-way ANOVA adjusting for intervention arm. The parent trial was not designed to have sufficient power to detect between-arm differences in vascular measures; 45 subjects in each intervention arm would provide a power of $8 \%$ to detect a between-arm difference of 0.005 $\mathrm{mm}$ in change in CIMT assuming a common standard deviation of $0.05 \mathrm{~mm}$, and a power of $22 \%$ to detect a between-arm difference of $0.05 \mathrm{~mm}$ in change in $\mathrm{AD}$ assuming a common standard deviation of 0.2 at $\mathrm{p}<0.05$. However, this study had more than $80 \%$ power to detect a Pearson correlation coefficient of 0.30 between BMI change and CIMT or AD change at $\mathrm{p}<0.05$.

Separate linear regression models were used to identify variablesassociated with 6 month changes in CIMT and AD. All cardiometabolic risk factors of interest were considered for inclusion in the models. The first multivariable model included intervention arm and when significant at $\mathrm{p}<0.10$ also included age, sex, race (black/non-black), and change in BMI. For the fully-adjusted multivariable models, additional variables with $P<0.20$ in simple correlations with CIMT or AD were considered. Each variable was added sequentially to the first multivariable model and variables with $P<0.10$ were kept in the final model. To evaluate the sensitivity of our results to the assumption of the missing data being missing completely at random, we evaluated linear mixed effects models for AD and CIMT. For the same covariates included in the multivariable linear regression models, baseline values and changes from baseline (for time-varying covariates) or the interaction of time with the baseline value (for time-invariant covariates) were included in the mixed models. All analyses were performed using SAS (version 9.2, SAS Institute, Cary, North Carolina). The level of statistical significance was set at a 2 -sided $P$-value of 0.05 .

\section{RESULTS}

CIMT and AD were measured at both time points in 90 participants. Key demographic and clinical characteristics are presented in Table 1 . There were no significant differences in baseline characteristics between intervention groups except that there were more men in the Diet+PA arm. Time spent in vigorous activity also showed a marginally significant difference between groups (Table 1).

Body weight, total body fat, waist circumference, and abdominal adipose content decreased in both intervention groups over the course of the study (Table 2). In the overall study sample, significant improvements were also seen in fasting glucose, insulin, homeostatic model assessment of insulin resistance, leptin, adiponectin, C-reactive protein, and AD. The Diet+PA arm showed a greater reduction in waist circumference than the Diet arm $(P=0.04)$, but other changes were not statistically significantly different between arms (Table 2 ). The increase in number of steps per day was marginally greater in the Diet+PA arm (Table 2); 12 
out of $41(29 \%)$ subjects in the Diet+PA arm averaged at least 10,000 steps/day at 6 months. Interestingly however, 8 out of $41(20 \%)$ of subjects in the Diet arm averaged at least 10,000 steps/day at 6 months, so some individuals in the Diet arm clearly chose to initiate PA before they were advised to do so. AD decreased more in men than women, and change in $\mathrm{AD}$ was positively correlated with age and changes in all measures of adiposity (Table 3). Change in CIMT was positively correlated with changes in BMI, weight, waist circumference, fat-free mass, leptin, and insulin. Neither CIMT change nor AD change was associated with changes in blood pressure or lipids, either in the total sample or in those not on medications affecting these respective measures.

Further analysis by BMI subgroup revealed that the most severely obese participants (baseline BMI $\geq 45 \mathrm{~kg} / \mathrm{m}^{2}$ ) experienced the numerically greatest decreases in CIMT and $\mathrm{AD}$, but these decreases were not statistically significantly different from those of less obese individuals ( $p>0.15$ for both).Individuals who lost $\geq 5 \%$ of their body weight experienced a significantreduction in mean CIMT $(-0.02 \mathrm{~mm}, P=0.002)$ whereas individuals who lost $<5 \%$ of their baseline body weight experienced a small increase in CIMT $(0.025 \mathrm{~mm}, P=0.02)$ (see Figure 1 in online supplement). Those subjects who achieved $\geq 5 \%$ weight loss were more likely to be in the Diet+PA arm (56\% vs. $44 \%, P=0.06)$.Importantly, the increase in CIMT in the group that achieved $<5 \%$ weight losswas influenced by one outlier, an individual with an increase in CIMT of $0.12 \mathrm{~mm}$ and a decrease in $\mathrm{AD}$ of $0.44 \mathrm{~mm}$. This individual may have experienced a decrease in CIMT after 6 months as the carotid artery had additional time to adapt to the large decrease in diameter. When this subject was removed from the analysis, the increase in CIMT in this subgroup was no longer statistically significant $(\mathrm{p}=0.07)$.

In the first multiple linear regression model, change in BMI was positively associated with change in CIMT after adjusting for intervention arm (Table 4). In the fully adjusted model, only change in fasting insulin was associated with 6 month change in CIMT (Table 4). Change in the homeostatic model assessment of insulin resistance showed a nearly identical association $(P=0.03)$ with change in CIMT when included in the model in place of insulin.

Different factors were found to explain the 6 month changes in AD and CIMT. In the first multivariable regression model for change in $\mathrm{AD}$, adjusting for intervention arm, age, andsex, change in BMI was a highly significant determinant of change in AD (Table 5). In the fully adjusted model, male sex and greater reductions in BMI and abdominal subcutaneous fat were associated with greater reductions in $\mathrm{AD}$ (Table 5). No significant interactions were detected in the fully adjusted models for either CIMT or AD.

As a sensitivity analysis, in linear mixed models for AD and CIMT, 19 subjects who had baseline but no 6 month carotid data were added to the analysis. Of all baseline measures, only age differed between these subjects ( 42.9 years, SD 6.1)and the 90 subjects with complete carotid data (47.1 years, SD 6.4) $(P=0.01)$. Results from the linear mixed models were similar to those from the linear regression models, with change in insulinshowing an association with CIMT change $(P=0.05)$ and changes in BMI $(P=0.02)$ and abdominal subcutaneous fat $(P=0.02)$ showing associations with AD change.

\section{DISCUSSION}

This study clearly shows that significant improvements in common carotid artery structure occur with an intensive lifestyle intervention in severely obese adults. The physical activity intervention promoted a greater reduction in waist circumference but did not significantly add to the reductions in CIMT or AD, perhaps because of the smaller than desired increase in activity in the Diet+PA arm. The approximately $8 \%$ weight loss overall resulted in a mean 
decrease in common carotid artery AD of $0.07 \mathrm{~mm}$. Though there was not a significant reduction in mean CIMT in the total sample, individuals who achieved at least $5 \%$ weight loss experienced a significant reduction in CIMT. Our results demonstrate that an intensive dietary intervention can significantly reverse some of the key adverse cardiometabolic and vascular structural changes brought about by excess weight in severely obese adults. However, the small average increase of 50 minutes in moderate to vigorous activity per week in the Diet+PA arm in this study was insufficient to add significantly to vascular changes over a 6 month time period.Importantly, data from a meta-analysis of 8 large prospective studies suggest that the mean CIMT reduction of $0.02 \mathrm{~mm}$ in study subjects who achieved at least 5\% weight loss can be expected to reduce incident myocardial infarction and stroke rates by $3 \%$ and $4 \%$ respectively in the long term. In addition, data from one large prospective study suggest that the overall mean $\mathrm{AD}$ reduction of $0.07 \mathrm{~mm}$ in this study can be expected to reduce incident coronary heart disease rates by $2 \%$ in men and $6 \%$ in women over the next 10 years. Thus, the improvements in common carotid artery geometry in this study may wellyieldvery meaningful long-term clinical benefits for these severely obese individuals.

In the severely obese, bariatric surgery generates marked improvements in structural and functional measures of atherosclerosis and arteriosclerosis . This invasive treatment option, however, can have numerous complications and cannot be recommended to all severely obese individuals. This is the first sizable study to report improvements in vascular structure with an intensive lifestyle intervention in severely obese adults, with decreases in carotid diameter being significant in the overall sample and decreases in CIMT being significant in those who achieved at least 5\% weight loss. These findings are similar to improvements in vascular structure and function seen with 5-10\% weight loss in overweight, class I, and class II obese adults. These improvements are substantially smaller than those seen in a small study $(n=14)$ of severely obese adults who experienced an average of $40 \%$ weight lossas a result of a highly intense exercise intervention (mean 3.3 hours of exercise per day) accompanied by moderate caloric restriction over 7 months. However, such an intense exercise intervention would be prohibitively expensive and time consuming to undertake on a large scale. Despite the reality that conventional therapy for severe obesity is often ineffective, intensive but widely manageable lifestyle interventions have been shown to be effective both in the present trial and a recent randomized trial comparing an intensive medical weight loss program in a primary care setting to usual care. The challenging key to the effectiveness of such interventions, however, is consistent and frequent contact with participants .

CIMT has been shown to regress or progress more slowly as cardiovascular risk factors improve with lifestyle modification . In this study, change in CIMT was correlated with changes in leptin, insulin, and several measures of body size. Because CIMT serves as a measure of both early atherosclerosis and vascular adaptation to changes in hemodynamics, increased CIMT could represent not only early atherosclerosis but also an adaptive response to the elevated cardiac output and blood flow that support increased metabolic needs in obese individuals . With the accumulation of excess weight, these hemodynamic alterations, along with concurrent hormonal and neural feedback, can lead to adverse arterial structural changes including increased diameter and intima-media thickening. It is interesting to note that changes in CIMT and AD showed different associations with cardiovascular and metabolic risk factors in this study, a finding that agrees with cross-sectional studies and suggests that distinct adaptive processes are represented by CIMT and AD .

Obese individuals have increased fat mass and fat-free mass, which together promote the aforementioned hemodynamic alterations. Fat-free mass is a strong independent correlate of left ventricular mass, CIMT, and CCA lumen diameter in cross-sectional studies . Excess 
adipose tissue alters circulating adipocytokine levels, such as leptin, which controls food intake and energy expenditure and increases with increasing body fat. Hyperleptinemia has been associated with greater oxidative stress and insulin resistance, both of whichpromote atherosclerosis. In this study however, the association between change in leptin and change in CIMT was explained by BMI change.

Insulin resistance and compensatory hyperinsulinemia can advance atherosclerosis by promoting interactions between circulating monocytes and the vascular endothelium and by enabling vascular smooth muscle cell migration and proliferation. Similar to this study, a recent study in obese adolescents found that a decrease in homeostatic model assessment of insulin resistance was independently associated with a reduction in CIMT during weight loss. Perhaps in severely obese individuals, the reduction in insulin resistance accompanying weight loss is key to the regression of early atherosclerosis and vascular maladaptation. Interestingly, individuals losing $<5 \%$ of their baseline body weight experienced an increase in CIMT. This could have been partly caused by a nonsignificant increase infasting insulin in this subgroup $(1.2 \mu \mathrm{U} / \mathrm{mL}, \mathrm{SD} 1.7)$, which was significantly different $(P=0.0001)$ from the decrease in insulin $(-6.6 \mu \mathrm{U} / \mathrm{mL}$, SD 1.0) in those achieving at least $5 \%$ weight loss. However, as noted, when one influential subject showing a large increase in CIMT was removed from the analysis, the increase in CIMT in those subjects who achieved $<5 \%$ weight loss was no longer statistically significant.

Elevated common carotid artery diameter is another structural marker of cardiovascular disease risk that is associated with numerous cardiovascular risk factors and has been shown to predict events .In this study, change in $\mathrm{AD}$ was positively correlated with changes in all measures of body size except fat-free mass, and change in abdominal subcutaneous fat was an independent correlate of change in AD. As with CIMT, this reflects the influence of excess body mass on hemodynamic changes leading to vascular adaptation. The distribution of adipose tissue is an important determinant of obesity-associated metabolic and cardiovascular complications. Abdominal visceral fat has been found to be a strong determinant of metabolic dysfunction and of structural and functional vascular alterations in a general population of older adults, class I and II obese adults, and severely obese adults . Although abdominal subcutaneous fat influences insulin resistance, it typically shows weaker associations with metabolic and vascular dysfunction than visceral fat both in the general population and in obese individuals. In severely obese individuals, however, total abdominal fat may be a more important determinant of cardiovascular risk than any abdominal fat compartment . The majority of body fat in obese adults is subcutaneous, thus abdominal subcutaneous fat is a stronger correlate of total abdominal fat and perhaps also of vascular structural alterations in the severely obese.

A major strength of this study was the frequent and structured contact during the intervention, which enabled many participants to achieve meaningful weight loss.One limitation was that only $11 \%$ of the participants in this study were men, which restricted our analyses of sex-specific responses. In addition, no participants in this study were diabetic, thus our findings are not generalizable to this large segment of the severely obese population. However, our results indicate the important role of insulin resistance in carotid intima-media thickening even in non-diabetic severely obese individuals. In addition, 40 participants in the parent randomized trial did not have carotid ultrasound scans at both time points, mainly due to scheduling difficulties. We detected no significant baseline differences between individuals with and without complete carotiddata in factors found to influence CIMT or AD changes (results not shown).Moreover, results from sensitivity analyses including individuals with baseline but no follow-up carotid data were very similar to those reported. The small sample size of this study certainly limited our power to detect differences by intervention arm or by subgroups of interest, particularly for the vascular 
changes, which showed considerable heterogeneity. Finally, no control group was used for the dietary intervention, which prevented us from establishing with certainty the causal effects of the dietaryintervention.

In conclusion, a 6-month intensive dietary intervention either with or without physical activity can result in clinically meaningful improvements in cardiometabolic risk factors and common carotid artery geometry in severely obese adults. Moreover, these data indicate that weight-loss induced reductions in insulin resistance and abdominal fat are particularly associated with improvements in vascular health, benefits that are most evident in the most severely obese.

\section{Supplementary Material}

Refer to Web version on PubMed Central for supplementary material.

\section{Acknowledgments}

We acknowledge Dr. Janice Sabatine, Avanti Strategies, Cranberry Township, Pennsylvania, for writing and editing assistance.

Sources of support This study was funded by the Commonwealth of Pennsylvania Department of Health, Harrisburg, Pennsylvania. Jennifer N. Cooper is supported by F31HL106986 from the National Heart, Lung, and Blood Institute of the National Institutes of Health, Bethesda, Maryland. Kelly J. Shields, Julius Asubonteng, and Michelle L. Meyer are supported byT32HL083825from the National Heart, Lung, and Blood Institute of the National Institutes of Health, Bethesda, Maryland.

\section{List of abbreviations}

$\begin{array}{ll}\text { AD } & \text { carotid inter-adventitial diameter } \\ \text { BMI } & \text { body mass index } \\ \text { CCA } & \text { common carotid artery } \\ \text { CIMT } & \text { carotid intima-media thickness } \\ \text { CT } & \text { Computed tomography } \\ \text { DXA } & \text { dual-energy x-ray absorptiometry } \\ \text { PA } & \text { physical activity }\end{array}$

\section{REFERENCES}

1. De Michele M, Panico S, Iannuzzi A, et al. Association of obesity and central fat distribution with carotid artery wall thickening in middle-aged women. Stroke. 2002; 33(12):2923-8. [PubMed: 12468792]

2. Zebekakis P, Nawrot T, Thijs L, et al. Obesity is associated with increased arterial stiffness from adolescence until old age. J Hypertens. 2005; 23(10):1839-46. [PubMed: 16148607]

3. Mavri A, Stegnar M, Sentocnik JT, et al. Impact of weight reduction on early carotid atherosclerosis in obese premenopausal women. Obes Res. 2001; 9(9):511-6. [PubMed: 11557831]

4. de las Fuentes L, Waggoner AD, Mohammed BS, et al. Effect of Moderate Diet-Induced Weight Loss and Weight Regain on Cardiovascular Structure and Function. J Am Coll Cardiol. 2009; 54(25):2376-81. [PubMed: 20082927]

5. Karason K, Wikstrand J, Sjostrom L, et al. Weight loss and progression of early atherosclerosis in the carotid artery: a four-year controlled study of obese subjects. Int J Obes Relat Metab Disord. 1999; 23(9):948-56. [PubMed: 10490801] 
6. Brethauer SA, Heneghan HM, Eldar S, et al. Early effects of gastric bypass on endothelial function, inflammation, and cardiovascular risk in obese patients. Surg Endosc. 2011; 25(8):2650-9.

[PubMed: 21416179]

7. Skilton MR, Sieveking DP, Harmer JA, et al. The effects of obesity and non-pharmacological weight loss on vascular and ventricular function and structure. Diabetes Obes Metab. 2008; 10(10): 874-84. [PubMed: 18034845]

8. Poirier P, Cornier MA, Mazzone T, et al. Bariatric surgery and cardiovascular risk factors: a scientific statement from the American Heart Association. Circulation. 2011; 123(15):1683-701. [PubMed: 21403092]

9. Goodpaster BH, Delany JP, Otto AD, et al. Effects of diet and physical activity interventions on weight loss and cardiometabolic risk factors in severely obese adults: a randomized trial. JAMA. 2010; 304(16):1795-802. [PubMed: 20935337]

10. Ryan DH, Johnson WD, Myers VH, et al. Nonsurgical weight loss for extreme obesity in primary care settings: results of the Louisiana Obese Subjects Study. Arch Intern Med. 2010; 170(2):14654. [PubMed: 20101009]

11. Lorenz MW, Markus HS, Bots ML, et al. Prediction of clinical cardiovascular events with carotid intima-media thickness: a systematic review and meta-analysis. Circulation. 2007; 115(4):459-67. [PubMed: 17242284]

12. Wildman RP, Schott LL, Brockwell S, et al. A dietary and exercise intervention slows menopauseassociated progression of subclinical atherosclerosis as measured by intima-media thickness of the carotid arteries. J Am Coll Cardiol. 2004; 44(3):579-85. [PubMed: 15358024]

13. Ahmadi N, Eshaghian S, Huizenga R, et al. Effects of intense exercise and moderate caloric restriction on cardiovascular risk factors and inflammation. Am J Med. 2011; 124(10):978-82. [PubMed: 21798505]

14. Eigenbrodt M, Sukhija R, Rose K, et al. Common carotid artery wall thickness and external diameter as predictors of prevalent and incident cardiac events in a large population study. Cardiovascular Ultrasound. 2007; 5(11):1476-7120.

15. Kozakova M, Palombo C, Paterni M, et al. Body composition and common carotid artery remodeling in a healthy population. J Clin Endocrinol Metab. 2008; 93(9):3325-32. [PubMed: 18593775]

16. Ben Driss A, Benessiano J, Poitevin P, et al. Arterial expansive remodeling induced by high flow rates. American Journal of Physiology - Heart and Circulatory Physiology. 1997; 272(2):H851H8.

17. Hahn C, Schwartz MA. The role of cellular adaptation to mechanical forces in atherosclerosis. Arterioscler Thromb Vasc Biol. 2008; 28(12):2101-7. [PubMed: 18787190]

18. Sarmento P, Plavnik F, Zanella M, et al. Association of Carotid Intima-media Thickness and Cardiovascular Risk Factors in Women Pre- and Post-bariatric Surgery. Obes Surg. 2009; 19(3): 339-44. [PubMed: 19083071]

19. Sturm W, Tschoner A, Engl J, et al. Effect of bariatric surgery on both functional and structural measures of premature atherosclerosis. Eur Heart J. 2009; 30(16):2038-43. [PubMed: 19502233]

20. Habib P, Scrocco JD, Terek M, et al. Effects of Bariatric Surgery on Inflammatory, Functional and Structural Markers of Coronary Atherosclerosis. Am J Cardiol. 2009; 104(9):1251-5. [PubMed: 19840571]

21. Jakicic JM, Marcus BH, Lang W, et al. Effect of exercise on 24-month weight loss maintenance in overweight women. Arch Intern Med. 2008; 168(14):1550-9. [PubMed: 18663167]

22. Pi-Sunyer X, Blackburn G, Brancati FL, et al. Reduction in weight and cardiovascular disease risk factors in individuals with type 2 diabetes: one-year results of the look AHEAD trial. Diabetes Care. 2007; 30(6):1374-83. [PubMed: 17363746]

23. Wing RR, Jakicic J, Neiberg R, et al. Fitness, fatness, and cardiovascular risk factors in type 2 diabetes: look ahead study. Med Sci Sports Exerc. 2007; 39(12):2107-16. [PubMed: 18046181]

24. Goodpaster BH, Kelley DE, Wing RR, et al. Effects of weight loss on regional fat distribution and insulin sensitivity in obesity. Diabetes. 1999; 48(4):839-47. [PubMed: 10102702] 
25. Matthews DR, Hosker JP, Rudenski AS, et al. Homeostasis model assessment: insulin resistance and beta-cell function from fasting plasma glucose and insulin concentrations in man. Diabetologia. 1985; 28(7):412-9. [PubMed: 3899825]

26. Njoroge JN, El Khoudary SR, Fried LF, et al. High urinary sodium is associated with increased carotid intima-media thickness in normotensive overweight and obese adults. Am J Hypertens. 2011; 24(1):70-6. [PubMed: 20508622]

27. Wendelhag I, Gustavsson T, Suurkula M, et al. Ultrasound measurement of wall thickness in the carotid artery: fundamental principles and description of a computerized analysing system. Clin Physiol. 1991; 11(6):565-77. [PubMed: 1769190]

28. Miyaki A, Maeda S, Yoshizawa M, et al. Effect of Weight Reduction With Dietary Intervention on Arterial Distensibility and Endothelial Function in Obese Men. Angiology. 2009; 60(3):351-7. [PubMed: 19022788]

29. Bots ML, Hofman A, Grobbee DE. Increased common carotid intima-media thickness. Adaptive response or a reflection of atherosclerosis? Findings from the Rotterdam Study. Stroke. 1997; 28(12):2442-7. [PubMed: 9412629]

30. Bella JN, Devereux RB, Roman MJ, et al. Relations of left ventricular mass to fat-free and adipose body mass: the strong heart study. The Strong Heart Study Investigators. Circulation. 1998; 98(23):2538-44. [PubMed: 9843460]

31. Czernichow S, Bertrais S, Oppert JM, et al. Body composition and fat repartition in relation to structure and function of large arteries in middle-aged adults (the SU.VI.MAX study). Int J Obes (Lond). 2005; 29(7):826-32. [PubMed: 15917850]

32. Beltowski J. Leptin and atherosclerosis. Atherosclerosis. 2006; 189(1):47-60. [PubMed: 16580676]

33. Wang CC, Goalstone ML, Draznin B. Molecular mechanisms of insulin resistance that impact cardiovascular biology. Diabetes. 2004; 53(11):2735-40. [PubMed: 15504952]

34. de Lima Sanches P, de Mello MT, Elias N, et al. Improvement in HOMA-IR is an independent predictor of reduced carotid intima-media thickness in obese adolescents participating in an interdisciplinary weight-loss program. Hypertens Res. 2011; 34(2):232-8. [PubMed: 21124323]

35. Crouse JR, Goldbourt U, Evans G, et al. Risk factors and segment-specific carotid arterial enlargement in the Atherosclerosis Risk in Communities (ARIC) cohort. Stroke. 1996; 27(1):6975. [PubMed: 8553406]

36. Steinke W, Els T, Hennerici M. Compensatory carotid artery dilatation in early atherosclerosis. Circulation. 1994; 89(6):2578-81. [PubMed: 8205667]

37. Fox CS, Massaro JM, Hoffmann U, et al. Abdominal visceral and subcutaneous adipose tissue compartments: association with metabolic risk factors in the Framingham Heart Study. Circulation. 2007; 116(1):39-48. [PubMed: 17576866]

38. Sutton-Tyrrell K, Newman A, Simonsick EM, et al. Aortic stiffness is associated with visceral adiposity in older adults enrolled in the study of health, aging, and body composition. Hypertension. 2001; 38(3):429-33. [PubMed: 11566917]

39. Tokita A, Ishigaki Y, Okimoto $\mathrm{H}$, et al. Carotid arterial elasticity is a sensitive atherosclerosis value reflecting visceral fat accumulation in obese subjects. Atherosclerosis. 2009; 206(1):168-72. [PubMed: 19296951]

40. Sturm W, Sandhofer A, Engl J, et al. Influence of visceral obesity and liver fat on vascular structure and function in obese subjects. Obesity (Silver Spring). 2009; 17(9):1783-8. [PubMed: 19325543]

41. Goodpaster BH, Thaete FL, Simoneau JA, et al. Subcutaneous abdominal fat and thigh muscle composition predict insulin sensitivity independently of visceral fat. Diabetes. 1997; 46(10):157985. [PubMed: 9313753]

42. Guzzaloni G, Minocci A, Marzullo P, et al. Sagittal abdominal diameter is more predictive of cardiovascular risk than abdominal fat compartments in severe obesity. Int J Obes (Lond). 2009; 33(2):233-8. [PubMed: 19139755] 
Table 1

Participant Characteristics at Baseline

\begin{tabular}{|c|c|c|c|c|}
\hline & Total $(\mathbf{n}=90)$ & Diet $(n=45)$ & Diet+PA $(n=45)$ & $\mathbf{P}$ \\
\hline Age (years) & $47.1(6.4)$ & $47.5(6.2)$ & $46.8(6.5)$ & 0.62 \\
\hline Male, n (\%) & $10(11.1)$ & $2(4.4)$ & $8(17.8)$ & 0.04 \\
\hline African American race, $\mathrm{n}(\%)$ & $29(32.2)$ & $16(35.6)$ & $13(28.9)$ & 0.50 \\
\hline Current smoker, n (\%) & $8(8.9)$ & $2(4.4)$ & $6(13.3)$ & 0.27 \\
\hline Blood pressure medication use, $\mathrm{n}(\%)$ & $35(38.9)$ & $15(33.3)$ & $20(44.4)$ & 0.28 \\
\hline Lipid lowering medication use, $\mathrm{n}(\%)$ & $13(14.4)$ & $7(15.6)$ & $6(13.3)$ & 0.76 \\
\hline \multicolumn{5}{|l|}{ Obesity severity, n (\%) } \\
\hline Class II (BMI $35-39.9 \mathrm{~kg} / \mathrm{m}^{2}$ ) & $25(27.8)$ & $12(26.7)$ & $13(28.9)$ & 0.81 \\
\hline Class III (BMI $\geq 40 \mathrm{~kg} / \mathrm{m}^{2}$ ) & $65(72.2)$ & $33(73.3)$ & $32(71.1)$ & \\
\hline BMI $\left(\mathrm{kg} / \mathrm{m}^{2}\right)$ & $43.9(5.7)$ & $44.0(6.6)$ & $43.8(4.8)$ & 0.87 \\
\hline Weight (kg) & $120.2(17.9)$ & $118.6(16.6)$ & $121.9(19.2)$ & 0.38 \\
\hline Waist circumference $(\mathrm{cm})$ & $123.0(12.6)$ & $121.2(12.6)$ & $124.8(12.6)$ & 0.18 \\
\hline Body fat $(\mathrm{kg})$ & $61.0(12.0)$ & $60.4(12.2)$ & $61.6(12.0)$ & 0.64 \\
\hline Fat free mass $(\mathrm{kg})$ & $58.0(8.9)$ & $57.0(7.1)$ & $59.0(10.4)$ & 0.29 \\
\hline Abdominal visceral fat $\left(\mathrm{cm}^{2}\right)^{a}$ & $200.3(71.0)$ & $186.9(62.9)$ & $213.7(76.7)$ & 0.08 \\
\hline Abdominal subcutaneous fat $\left(\mathrm{cm}^{2}\right)^{a}$ & $740.4(180.6)$ & $739.3(187.2)$ & $741.5(175.8)$ & 0.96 \\
\hline Systolic blood pressure $(\mathrm{mm} \mathrm{Hg})$ & $135.2(12.6)$ & $133.4(16.6)$ & $135.9(11.0)$ & 0.61 \\
\hline Diastolic blood pressure (mm Hg) & $77.2(8.1)$ & $76.6(8.5)$ & $77.8(7.9)$ & 0.51 \\
\hline Glucose $(\mathrm{mmol} / \mathrm{L})$ & $5.2(0.6)$ & $5.2(0.6)$ & $5.2(0.6)$ & 0.92 \\
\hline Insulin $(\mathrm{pmol} / \mathrm{L})^{b}$ & $109.7(63.9)$ & $104.2(59.7)$ & $115.3(68.8)$ & 0.42 \\
\hline $\begin{array}{l}\text { Homeostatic model assessment of insulin resistance } \\
(\mathrm{mmol} / \mathrm{L} \times \mu \mathrm{U} / \mathrm{mL})^{b}\end{array}$ & $3.7(2.3)$ & $3.5(2.1)$ & $3.9(2.6)$ & 0.42 \\
\hline Leptin $(\mu \mathrm{g} / \mathrm{L})^{b}$ & $53.7(22.4)$ & $55.2(21.6)$ & $52.4(23.3)$ & 0.55 \\
\hline Adiponectin $(\mu \mathrm{g} / \mathrm{mL})^{c}$ & $3.9(1.9)$ & $4.3(2.0)$ & $3.6(1.8)$ & 0.14 \\
\hline Triglycerides $(\mathrm{mmol} / \mathrm{L})$ & $1.2(1.0,1.7)$ & $1.3(1.0,1.5)$ & $1.2(0.9,1.8)$ & 0.82 \\
\hline Total cholesterol $(\mathrm{mmol} / \mathrm{L})$ & $4.9(0.8)$ & $5.0(0.7)$ & $4.9(0.9)$ & 0.80 \\
\hline High density lipoprotein cholesterol ( $\mathrm{mmol} / \mathrm{L})$ & $1.2(0.3)$ & $1.3(0.3)$ & $1.2(0.3)$ & 0.34 \\
\hline Low density lipoprotein cholesterol $(\mathrm{mmol} / \mathrm{L})$ & $3.2(0.7)$ & $3.2(0.7)$ & $3.2(0.8)$ & 0.98 \\
\hline C-reactive protein (mg/L) & $6.9(3.4,11.3)$ & $5.5(3.3,11.8)$ & $8.2(4.4,10.6)$ & 0.34 \\
\hline Number of steps per day ${ }^{d}$ & $7411.4(2965.7)$ & $7340.1(2861.5)$ & $7479.5(3092.8)$ & 0.83 \\
\hline Moderate activity (min/week) ${ }^{d}$ & $604.8(440.6)$ & $605.6(485.5)$ & $602.3(398.6)$ & 0.97 \\
\hline Vigorous activity (min/week) ${ }^{d}$ & $28.6(11.3,63.4)$ & $22.8(9.2,48.3)$ & $31.1(17.6,82.3)$ & 0.05 \\
\hline Mean CIMT (mm) & $0.77(0.10)$ & $0.77(0.11)$ & $0.77(0.10)$ & 0.91 \\
\hline Mean AD (mm) & $7.14(0.61)$ & $7.12(0.60)$ & $7.16(0.63)$ & .76 \\
\hline
\end{tabular}

Values are mean (SD), median (25th, 75th percentiles), or n (\%); P-values are from independent samples t-test or Mann-Whitney U test for continuous variables; p-value by chi-square test or Fisher's Exact test for categorical variables

${ }_{\mathrm{n}=}^{a} 44$ Diet, 44 Diet+PA

$b_{\mathrm{n}=43}$ Diet;

Metabolism. Author manuscript; available in PMC 2013 November 01. 
$c_{\mathrm{n}=36}$ Diet, 41 Diet+PA

$d_{\mathrm{n}=43}$ Diet, 45 Diet+PA; $\mathrm{AD}=$ common carotid artery inter-adventitial diameter; $\mathrm{BMI}=$ body mass index; $\mathrm{CIMT}=$ common carotid artery intimamedia thickness; $\mathrm{PA}=$ physical activity 


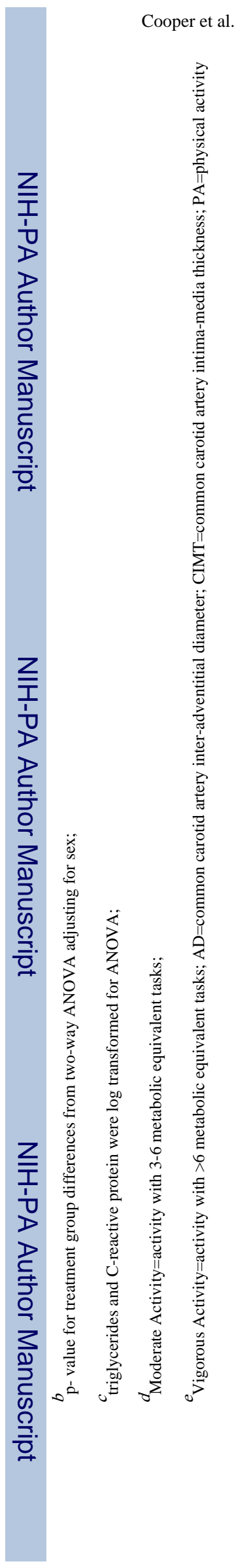

Page 15

Metabolism. Author manuscript; available in PMC 2013 November 01. 
Table 3

Pearson Correlations between common carotid artery structural changes and demographic variables or changes in cardiometabolic factors

\begin{tabular}{|c|c|c|}
\hline Variable & Change in AD & Change in CIMT \\
\hline \multicolumn{3}{|l|}{ Baseline demographic variables } \\
\hline Age & $0.23^{*}$ & -0.13 \\
\hline Male sex & $-0.24^{*}$ & 0.02 \\
\hline African American race & 0.002 & 0.09 \\
\hline \multicolumn{3}{|l|}{ Six month change variables } \\
\hline Inter-adventitial diameter & ---- & 0.10 \\
\hline Weight & $0.32^{*}$ & $0.25^{*}$ \\
\hline Body mass index & $0.31^{*}$ & $0.23^{*}$ \\
\hline Waist circumference & $0.23^{*}$ & $0.24^{*}$ \\
\hline Systolic blood pressure & -0.06 & -0.19 \\
\hline Diastolic blood pressure & 0.02 & -0.17 \\
\hline Glucose & 0.06 & 0.03 \\
\hline High density lipoprotein cholesterol & 0.09 & -0.15 \\
\hline Low density lipoprotein cholesterol & 0.08 & -0.04 \\
\hline Triglycerides & 0.18 & 0.11 \\
\hline Number of steps/day & -0.13 & -0.02 \\
\hline Moderate Activity min/week & -0.09 & 0.05 \\
\hline Vigorous Activity min/week & -0.09 & 0.006 \\
\hline Abdominal Visceral Fat & $0.24^{*}$ & 0.14 \\
\hline Abdominal Subcutaneous Fat & $0.35^{*}$ & 0.10 \\
\hline Body fat mass & $0.39^{*}$ & 0.13 \\
\hline Fat free mass & 0.15 & $0.31^{*}$ \\
\hline Adiponectin & 0.13 & -0.02 \\
\hline C-reactive protein & 0.07 & 0.11 \\
\hline Leptin & 0.07 & $0.22^{*}$ \\
\hline Insulin & 0.19 & $0.30^{*}$ \\
\hline
\end{tabular}




\section{Table 4}

Multivariable Linear Regression Models for 6 Month Change in Common Carotid Artery Intima-Media Thickness

\begin{tabular}{|c|c|c|c|c|}
\hline Model & Variable & $\begin{array}{c}\text { Parameter } \\
\text { estimate }\end{array}$ & s.e & $\mathbf{P}$ \\
\hline \multicolumn{5}{|c|}{ Model $1\left(\mathbf{R}^{2}=0.06\right)^{a}$} \\
\hline & Diet Arm & 0.0024 & 0.012 & 0.84 \\
\hline & Change in BMI $\left(\mathrm{kg} / \mathrm{m}^{2}\right)^{b}$ & 0.0058 & 0.003 & 0.03 \\
\hline \multicolumn{5}{|c|}{ Model $2\left(\mathrm{R}^{2}=0.11\right)^{c}$} \\
\hline & Diet Arm & 0.0046 & 0.012 & 0.70 \\
\hline & Change in BMI $\left(\mathrm{kg} / \mathrm{m}^{2}\right)^{b}$ & 0.0032 & 0.003 & 0.45 \\
\hline & Change in Insulin $(\mu \mathrm{U} / \mathrm{mL})^{b}$ & 0.0017 & 0.0008 & 0.03 \\
\hline \multicolumn{5}{|c|}{ Results are from ordinary least squares regression with the listed independent variables; } \\
\hline \multicolumn{5}{|c|}{${ }^{a}$ Model $1 \mathrm{n}=90$} \\
\hline
\end{tabular}


Table 5

Multivariable Linear Regression Models for 6 Month Change in Common Carotid Artery Inter-Adventitial Diameter

\begin{tabular}{|c|c|c|c|c|}
\hline Model & Variable & $\begin{array}{l}\text { Parameter } \\
\text { estimate }\end{array}$ & s.e & $\mathbf{P}$ \\
\hline \multicolumn{5}{|c|}{ Model $1\left(R^{2}=0.19\right)^{a}$} \\
\hline & Diet Arm & 0.01 & 0.04 & 0.80 \\
\hline & Age (years) & 0.006 & 0.003 & 0.07 \\
\hline & Male sex & -0.14 & 0.06 & 0.04 \\
\hline & Change in BMI $\left(\mathrm{kg} / \mathrm{m}^{2}\right) b$ & 0.03 & 0.009 & 0.002 \\
\hline \multicolumn{5}{|c|}{ Model $2\left(\mathbf{R}^{2}=0.25\right)^{c}$} \\
\hline & Diet Arm & 0.003 & 0.04 & 0.95 \\
\hline & Male sex & -0.16 & 0.06 & 0.01 \\
\hline & Age (years) & 0.005 & 0.003 & 0.15 \\
\hline & Change in BMI $\left(\mathrm{kg} / \mathrm{m}^{2}\right)^{b}$ & 0.02 & 0.01 & 0.03 \\
\hline & $\begin{array}{l}\text { Change in Abdominal subcutaneous } \\
\text { fat }\left(\mathrm{cm}^{2}\right) b\end{array}$ & 0.0005 & 0.0002 & 0.04 \\
\hline \multicolumn{5}{|c|}{ Results are from ordinary least squares regression with the listed independent variables; } \\
\hline \multicolumn{5}{|c|}{ a Model $1 \mathrm{n}=90$} \\
\hline \multicolumn{5}{|c|}{$b_{\mathrm{BMI}}$ and abdominal subcutaneous fat were evaluated as changes from baseline; } \\
\hline
\end{tabular}

Metabolism. Author manuscript; available in PMC 2013 November 01. 